\title{
El smartphone desde la perspectiva docente: ¿una herramienta de tutorización o un catalizador de ciberacoso?
}

\section{The smartphone from teachers' perspective: a guiding tool or a cyberbullying catalyst?}

\author{
Bernardo Tabuenca \\ Universidad Politécnica de Madrid. Madrid, España \\ bernardo.tabuenca@upm.es \\ Juan-José Sánchez-Peña \\ Universidad de Alcalá. Alcalá de Henares, España \\ juanjo.sanchez@uah.es \\ María-José Cuetos-Revuelta \\ Universidad Internacional de la Rioja. Logroño, España \\ mariaj.cuetos@gmail.com
}

\begin{abstract}
Resumen
La era de los teléfonos móviles inteligentes cumple una década de madurez y las instituciones educativas parecen no asimilar los cambios que impone la sociedad digital. La necesidad de articular métodos de tutorización con ayuda de tecnología popular no es nueva, sino que es un agregado a barreras precedentes con tecnologías como Internet o las redes sociales. En este contexto, el ciberacoso representa una de las principales razones del desajuste con el cual profesores y dirigentes lidian para armonizar el uso de dispositivos móviles en los centros educativos. Este artículo explora desde los ojos de 135 profesores $(M=32)$ qué uso se hace del móvil con fines educativos, su predisposición a utilizar móviles propios y redes sociales como herramienta de tutorización, qué peligros y ventajas se identifican de su uso, qué regulación existe actualmente en los centros educativos, y qué edad consideran apropiada para entregar un móvil con Internet a un niño/adolescente. Los resultados de este trabajo dan evidencia de los riesgos de la precocidad en el uso de dispositivos móviles, y se sugieren claves para facilitar la tutorización con ayuda de dispositivos móviles. Este trabajo ofrece una base de conocimiento relevante a futuras iniciativas de establecer usos apropiados del móvil desde la perspectiva docente en España.
\end{abstract}

Palabras clave: m-learning, adolescencia, cultura digital, aprendizaje ubicuo, políticas educativas

\begin{abstract}
The mobile era turns one decade of maturity but educational institutions do not seem to assimilate changes imposed by digital society. The need to assemble tutoring methods supported with popular technology is not new, but rather an added issue over preceding pitfalls with technologies like Internet or social networks. In this context, cyberbullying represents one of the main reasons for this discrepancy where teachers and educational directors struggle to harmonize the use of mobile devices in schools. This manuscript explores from the perspective of 135 teachers $(M=32)$ how mobiles are used for learning, their inclination with regard to use own devices and social networks for tutoring students, threats and opportunities of mobile devices, which regulation they know with regard to the use of mobile devices in schools, and the age they consider suitable for children to own a smartphone. The results not only provide evidence of the risks in immaturedelivery of smartphone to a child/adolescent, but also show lessons learned to facilitate tutoring supported with mobile technology. Overall this work provides a base of relevant knowledge for future research to assemble suitable use of mobiles from teachers' perspective in Spain.
\end{abstract}

Keywords: m-learning, adolescence, digital culture, ubiquitous learning, educational policy 
RED. Revista de Educación a Distancia. Núm. 59, Artíc. 01, 30-04-2019

DOI: http://dx.doi.org/10.6018/red/59/01

\section{Introducción}

Los frecuentes casos de ciberacoso y su impacto social han llevado a buena parte de los centros educativos a prohibir el uso de los dispositivos móviles en sus instalaciones e incluso impedir que sean introducidos en clase. Esta situación topa con la realidad actual en la que es común utilizar dispositivos móviles propios o Bring Your Own Device (BYOD) para acceder a recursos de aprendizaje desde la escuela, desde el trabajo y desde casa (Johnson, Adams Becker, Estrada, \& Freeman, 2015; Tabuenca, Drachsler, Ternier, \& Specht, 2012).

La adopción del mobile learning en espacios de educación superior es ya un hecho (Adam Becker et al., 2017) que se está viendo impulsado por la conveniencia de los dispositivos móviles para la comunicación entre docentes y discentes, para la compartición de contenidos o para llevar una trazabilidad del proceso de aprendizaje (Kukulska-Hulme \& Traxler, 2005; Sharples, Taylor, \& Vavoula, 2007; Tabuenca et al., 2012; Tabuenca, Kalz, Drachsler, \& Specht, 2015; Tabuenca, Ternier, \& Specht, 2013; Tabuenca, Verpoorten, \& Specht, 2013). Sin embargo, el uso de las nuevas tecnologías puede acarrear conductas de riesgo que requieren un uso con garantías de seguridad en edad escolar (Cerezo-Ramirez, 1997; Garaigordobil, 2011). El ciberacoso es una conducta agresiva e intencional que se repite de forma frecuente en el tiempo mediante el uso por parte de un individuo (o grupo), de dispositivos electrónicos sobre una víctima que no puede defenderse por sí misma fácilmente (Smith et al., 2008). Las herramientas disponibles en Internet en general, y los móviles en particular ayudan a la propagación de ese mal comportamiento. Las vías utilizadas para realizar ciberacoso abarcan mensajes de texto, llamadas directas, fotografías o videos de vejaciones que se difunden vía móvil en redes sociales (Giménez-Gualdo, Maquilón-Sánchez, \& Arnaiz Sánchez, 2014; Hosseinmardi, Rafiq, Han, Lv, \& Mishra, 2016; Mercedes Durán, 2015).

El ciberacoso en los últimos años sigue una tendencia creciente en número de acosados (ANAR \& MutuaMadrileña, 2017; Garmendia Larrañaga, Jiménez Iglesias, Casado, \& Marcheroni, 2016) y a diferencia de la era pre-Internet en el que el hostigamiento cesaba fuera del centro escolar o en el entorno familiar de la víctima, en la era móvil el acoso también crece en continuidad ya que es ubicuo en el espacio y en el tiempo (CerezoRamirez, 2012).

El enfoque de los profesores acerca de los buenos y malos usos del Smartphone en la educación es primordial ya que en muchas ocasiones los docentes pasan más tiempo con los adolescentes que sus propios padres. El trabajo de (O'Bannon \& Thomas, 2015) examina las percepciones de 245 aspirantes a profesor de Estados Unidos acerca de cómo pueden ayudar los móviles en el aula y cuales son los principales obstáculos para utilizarlos. Los resultados concluyen un $45 \%$ de participantes a favor del uso de teléfonos móviles en el aula. Las funcionalidades mejor valoradas fueron el acceso a Internet, los sistemas de respuesta automáticos, y la aplicaciones educacionales. Contrariamente, identificaron como principales barreras su facilidad para "hacer trampas" no controlables por el profesorado, la distracción durante las tareas de aprendizaje, y el cyberbullying. Estudios análogos con profesores y resultados similares se han realizado en Israel (Eden, Heiman, \& OlenikShemesh, 2013), Turquía (Ryan, Kariuki, \& Yilmaz, 2011), Taiwán (Huang \& Chou, 2013) o Canadá (Li, 2008). El trabajo de (Brazuelo-Grund, Gallego-Gil, \& Cacheiro-González, 2017) pone en evidencia el gran desconocimiento de los docentes españoles en cuanto a los usos educativos del teléfono móvil como herramienta de enseñanza y aprendizaje (93,3\%).

El presente trabajo explora qué oportunidades y riesgos conlleva el uso de los móviles desde la perspectiva docente en España. Para ello se plantean las siguientes preguntas de investigación:

1. ¿Qué uso hacen los docentes de sus dispositivos móviles con fines educativos? ¿Usan Smartphone y/o tableta? ¿Qué herramientas móviles utilizan en su actividad docentes y para qué?

2. ¿Cuál es la predisposición de los docentes para utilizar móviles (propios) como herramienta de tutorización?

3. ¿Cuál es la predisposición de los docentes a utilizar las redes sociales para comunicarse con los alumnos?

4. ¿Cuáles son los principales riesgos de utilizar el móvil en los centros escolares desde la perspectiva docente?.

5. ¿Cuáles son las principales ventajas de utilizar el móvil en los centros escolares desde la perspectiva docente?

El smartphone desde la perspectiva docente: ¿una herramienta de tutorización o un catalizador de ciberacoso? Bernardo Tabuenca, Juan-José Sánchez-Peña y María-José Cuetos-Revuelta.

Página 2 de 14 
RED. Revista de Educación a Distancia. Núm. 59, Artíc. 01, 30-04-2019

DOI: http://dx.doi.org/10.6018/red/59/01

El uso de los dispositivos móviles en el aula está regulado por las normas particulares de cada profesor, centro educativo, comunidad autónoma y país. El estudio comparativo de (Cerezo Ramírez \& Rubio-Hernández, 2017) da a conocer cómo se está regulando la problemática del ciberacoso en Decretos, Órdenes, Resoluciones que aplican a cada comunidad autónoma. En este trabajo se estudia en qué protocolos se traducen las directrices redactadas en estos documentos normativos desde la visión docente, y cuál es su opinión sobre qué organismo debería regular su uso. Para ello se plantea la siguiente pregunta de investigación:

6. ¿Qué regulación existe actualmente con respecto al uso de dispositivos móviles en los centros educativos? ¿Qué figura debería regular el uso de los dispositivos móviles en los centros educativos?

Estadísticas recientes indican que el $51 \%$ de los niños españoles de 11 años ya dispone de un móvil, una proporción que crece hasta el 94\% entre los de 15 años (INE, 2016). El debate sobre cuál es la edad apropiada para entregar un móvil a un niño varia en función de cada entorno familiar, de la misma forma que parece haber una relación entre conductas de acoso escolar y condiciones sociofamiliares (Ortega Barón, Jessica; Buelga, Sofía; Cava, 2016; Prodócimo, Cerezo, \& Arense, 2014). En este trabajo, se traslada la valoración a los docentes, y además se explora la correlación entre la edad del profesor y la edad sugerida para el uso de teléfonos móviles. Para ello se plantea la siguiente pregunta de investigación:

7. ¿Cuál es la edad apropiada para entregar un móvil con Internet a un niño desde la perspectiva docente? ¿Existe alguna correlación entre la edad de profesor con la edad propuesta para el uso de teléfonos móviles?

\section{Método}

Este estudio se lleva a cabo en el contexto de los estudios de posgrado en un Máster Oficial Universitario en eLearning y Tecnología Educativa de una universidad española. Como parte de las actividades en la asignatura "Gestión de Alumnos, Sistemas Tutoriales, Evaluación y Redes Sociales en el Aprendizaje", se invitaba a los estudiantes a debatir en el foro de la plataforma, y a participar en un cuestionario acerca de los riesgos y ventajas de utilizar los dispositivos móviles en la actividad docente y discente. El sistema recogió respuestas entre marzo y julio del 2017.

\subsection{Participantes}

De los 151 alumnos matriculados en el máster, el 90\% ( $n=135)$ accedieron a participar en el estudio aceptando el consentimiento informado que se incluyó en el propio formulario. Los participantes en este estudio son profesores $(98.51 \%)$ o aspirantes a profesor (1.49\%). El 65\% eran mujeres y la edad media de los profesores participantes es de 32.45 años con una desviación estándar de 6.06. La edad media de sus alumnos es de 11.5 años con una desviación estándar de 6.32 . El 94\% de los profesores reportaron haber impartido docencia en modalidad presencial, el $9 \%$ en la modalidad online (eLearning), y el $6 \%$ en la modalidad mixta que combina los dos anteriores (blended learning).

La Tabla 1 especifica en qué nivel educativo imparten clase, la comunidad autónoma en la que ejercen como docentes actualmente, las asignaturas y la modalidad en las que imparten o han impartido a lo largo de su carrera. Todos los profesores encuestados reportaron tener un móvil con conexión a Internet.

El smartphone desde la perspectiva docente: ¿una herramienta de tutorización o un catalizador de ciberacoso? Bernardo Tabuenca, Juan-José Sánchez-Peña y María-José Cuetos-Revuelta.

Página 3 de 14 
RED. Revista de Educación a Distancia. Núm. 59, Artíc. 01, 30-04-2019

DOI: http://dx.doi.org/10.6018/red/59/01

Tabla 1. Profesores participantes en el estudio

\begin{tabular}{lcc}
\hline Docente de & $\mathbf{N}(\mathbf{1 3 5})$ & $\%$ \\
\hline Primaria & 67 & 50 \\
Secundaria & 45 & 33.58 \\
Otros (Academias, centros privados ...) & 10 & 7.46 \\
Formación profesional & 9 & 6.71 \\
No ejerce como profesor actualmente & 2 & 1.49 \\
Estudios universitarios & 1 & 0.74 \\
\hline Materia & & \\
\hline Matemáticas & 60 & 18.18 \\
Lengua y literatura & 56 & 16.97 \\
Otras ... & 49 & 14.85 \\
Idiomas & 31 & 9.39 \\
Educación física & 27 & 8.18 \\
Geografía e historia & 25 & 7.58 \\
Educación plástica & 24 & 7.27 \\
Biología y geología & 15 & 4.55 \\
Música & 12 & 3.64 \\
Informática & 10 & 3.03 \\
Física y química & 6 & 1.82 \\
Tecnología & 6 & 1.82 \\
Religión & 5 & 1.52 \\
NA & 4 & 1.21 \\
\hline
\end{tabular}

\begin{tabular}{lcc}
\hline Comunidad autónoma & N(135) & $\%$ \\
\hline Aragón & 28 & 20.90 \\
Andalucía & 23 & 17.16 \\
Comunidad Valenciana & 18 & 13.43 \\
Extremadura & 15 & 11.19 \\
Región de Murcia & 11 & 8.21 \\
Comunidad de Madrid & 8 & 5.97 \\
Asturias & 7 & 5.22 \\
Galicia & 6 & 4.48 \\
Castilla La Mancha & 5 & 3.73 \\
Fuera de España & 4 & 2.99 \\
Canarias & 2 & 1.49 \\
Castilla y León & 2 & 1.49 \\
Comunidad Foral de Navarra & 2 & 1.49 \\
Ciudad autónoma de Melilla & 1 & 0.75 \\
La Rioja & 1 & 0.75 \\
País Vasco & 1 & 0.75 \\
\hline
\end{tabular}

\subsection{Materiales y diseño del estudio}

El debate se propuso mediante un hilo de discusión online en el cual los alumnos participaban exponiendo sus puntos de vista acerca de un tema específico. Primeramente se contextualizó la pregunta de investigación, focalizando en la precocidad progresiva en el uso de teléfonos móviles por parte de los niños españoles referenciando la encuesta sobre equipamiento y uso de tecnologías de información y comunicación en los hogares (INE 2016). Se distribuyó un enlace al cuestionario para que pudieran completarlo sin restricciones de tiempo.

\subsection{Instrumentos de medida}

El cuestionario incluyó preguntas abiertas y Likert-scales de 5 elementos (Likert, 1932), para que valorasen en qué grado estaban de acuerdo con determinadas sentencias, preguntas de respuesta numérica, preguntas de selección simple, y preguntas de selección múltiple (Tabla 2).

Tabla 2. Preguntas de investigación e instrumentos de medida.

\begin{tabular}{cc}
\hline ID & Descripción \\
\hline & PI1. Uso de los dispositivos móviles en la actividad docente. \\
\hline C1 & ¿Utilizas el móvil para tu actividad docente? \\
C2 & ¿Utilizas tableta para tu actividad docente? \\
C3 & Por favor, describe que herramientas móviles utilizas en tu actividad docente, y para qué: \\
\hline & PI2. Predisposición a utilizar móviles. \\
\hline C4 & ¿Estarías dispuesto a utilizar tu teléfono móvil PERSONAL como herramienta para tutorizar a los alumnos? \\
C5 & ¿Estarías dispuesto a utilizar un teléfono móvil CORPORATIVO (de tu colegio, instituto o universidad...) como herramienta para \\
& tutorizar a los alumnos? \\
\hline \multicolumn{2}{c}{ PI3. Predisposición a utilizar las redes sociales para comunicarse con los alumnos. } \\
\hline C6 & ¿Estarías dispuesto a utilizar estas redes sociales para comunicarte con los alumnos? \\
\hline C7 & ¿Consideras importantes los siguientes RIESGOS de utilizar el móvil en los centros escolares? \\
\hline & PI5. Ventajas de utilizar móvil en los centros escolares. \\
\hline C8 & ¿Consideras importantes las siguientes VENTAJAS de utilizar móvil en los centros escolares? \\
\hline & PI6. Regulación del uso de móviles. \\
\hline C9 & ¿Qué regulación del uso del móvil se aplica en el centro educativo en el que impartes clase? \\
C10 & Actualmente no hay una normativa general sobre el uso del móvil en los centros escolares ¿Quién consideras que debería \\
\hline & regularlo? \\
\hline C11 & Por tu experiencia como docente ¿Cuál consideras que es la edad apropiada para dar un teléfono con Internet a un niño? \\
\hline
\end{tabular}

El smartphone desde la perspectiva docente: ¿una herramienta de tutorización o un catalizador de ciberacoso? Bernardo Tabuenca, Juan-José Sánchez-Peña y María-José Cuetos-Revuelta.

Página 4 de 14 
RED. Revista de Educación a Distancia. Núm. 59, Artíc. 01, 30-04-2019

DOI: http://dx.doi.org/10.6018/red/59/01

\subsection{Análisis de los datos}

Los datos de cuestionario fueron importados desde la plataforma de encuestas a formato MS Excel. Posteriormente fueron exportados a formato CSV para analizarlos con R Studio (v0.98.1102)

\section{Resultados}

En esta sección se presentan los resultados de la mediciones listadas en la Tabla 2 clasificadas por preguntas de investigación (PI1-PI7):

\subsection{El móvil en la docencia}

En la primera pregunta de investigación se pretendía explorar qué uso hacen los docentes de sus dispositivos móviles con fines educativos, qué herramientas móviles utilizan en su actividad docente, y para qué utilizan estas herramientas. En $\mathrm{C} 1$ el $99.25 \%$ de los profesores indicaron tener un dispositivo móvil con conexión a Internet. El 77,42\% de los profesores indicaron utilizar su móvil personal en su actividad docente. En C2, el 51\% de los profesores reportaron utilizar una tableta digital en su actividad docente.

En C3 se pedía describir qué herramientas móviles utilizaban en su actividad docente y con qué propósito mediante una pregunta de texto libre. En la Figura 1 se ilustra mediante una tag cloud los conceptos más citados en correspondencia con el tamaño con el que se representan.

\section{aplicaciones app aprendizase asistencia bussureda \\ calificaciones classdojo companeros compartir comunicar contenidos control correo-electronico crear cuaderno desarrollo dispositivos docente edmodo educativo} equipo familias google google-classroom google-drive herramientas idoceo internet iseneca juegos kahoot lista MOVil moviles notas ordenador padres pizarra-digital plataforma portatil presentaciones profesor recursos socrative tablet tareas trabajo videos whatsapp youtube

El smartphone desde la perspectiva docente: ¿una herramienta de tutorización o un catalizador de ciberacoso? Bernardo Tabuenca, Juan-José Sánchez-Peña y María-José Cuetos-Revuelta.

Página 5 de 14 
RED. Revista de Educación a Distancia. Núm. 59, Artíc. 01, 30-04-2019

DOI: http://dx.doi.org/10.6018/red/59/01

Figura 1. Herramientas móviles y su propósito en la actividad docente.

\subsection{Predisposición docente al uso de dispositivos móviles para la tutorización}

En la segunda pregunta de investigación se exploraba cuál es la predisposición de los docentes para utilizar los móviles como herramienta de tutorización. Además, se buscaba cuantificar en qué medida estarían dispuestos a realizar tareas de tutorización utilizando su propio dispositivo móvil.

En cuanto al uso del móvil personal como herramienta de soporte a la tutorización docente (Figura 2), el $29.85 \%$ indicaron "Sí, siempre", el $42.53 \%$ indicaron "Sí, pero sólo durante la jornada laboral y periodo lectivo", y el $27.61 \%$ indicaron no estar predispuestos a usarlo. Por otro lado, cuando se les preguntó por su predisposición a utilizar un móvil corporativo como herramienta de soporte a la tutorización docente, el $46.26 \%$ indicaron "Sí, siempre", el 49.25\% indicaron "Sí, pero sólo durante la jornada laboral y periodo lectivo", y el 4.47 indicaron no estar predispuestos a uso.

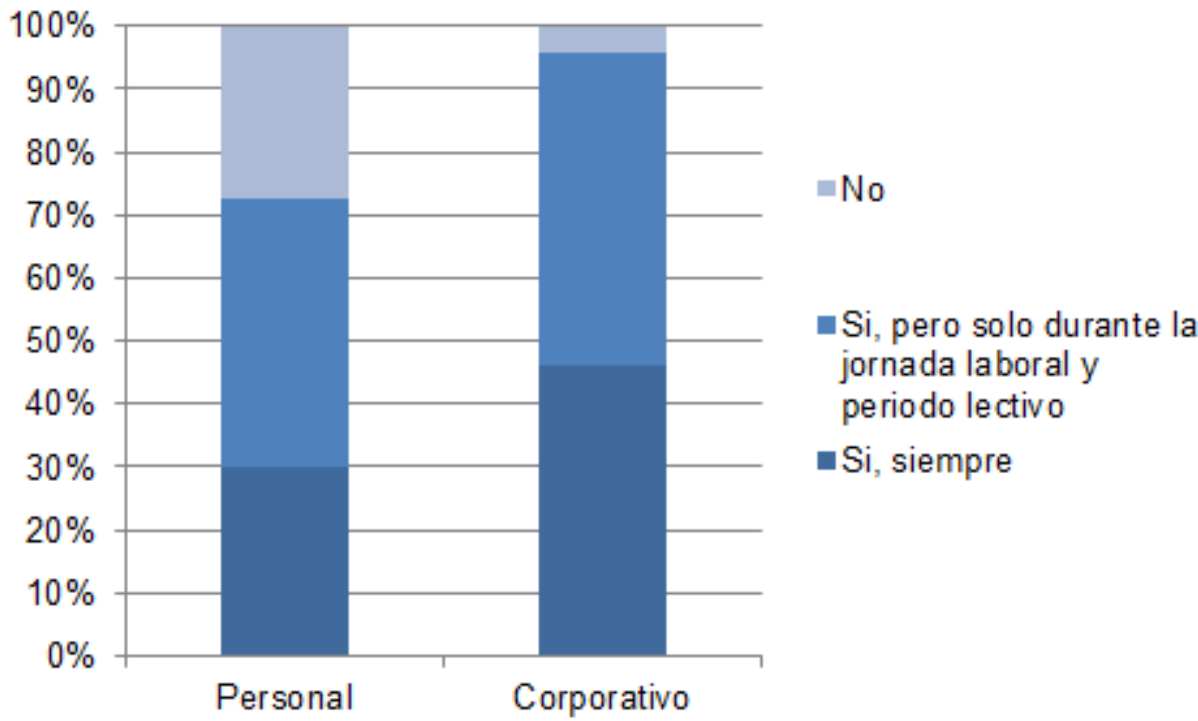

Figura 2. Predisposición a utilizar teléfono móvil personal/corporativo como herramienta para tutorizar a los alumnos.

\subsection{Predisposición docente al uso de las redes sociales para comunicarse con el alumnado}

En la tercera pregunta de investigación se explora la predisposición de los docentes al uso de redes sociales para comunicarse con los alumnos. Según se indica en la Tabla 3, los resultados revelan que los profesores estuvieron más predispuestos a unas redes sociales que a otras, identificando YouTube $(M=4.43 ; S D=0.68)$, Google+ $(M=3.98 ; S D=1.00)$, y Pinterest $(M=3.86 ; S D=0.96)$ como las principales. De las redes sociales citadas, los profesores reportaron una menor predisposición a utilizar Tuenti $(M=2.23 ; S D=1.17)$, Instagram $(\mathrm{M}=2.58 ; \mathrm{SD}=1.29)$ y Facebook $(\mathrm{M}=2.81 ; \mathrm{SD}=1.37)$. La valoración media con respecto a todas las redes sociales fue de $M=3.17(S D=1.35)$.

Tabla 3. Predisposición docente a utilizar las redes sociales para comunicarse con los alumnos. N=135(\%)

\begin{tabular}{|c|c|c|c|c|c|c|c|}
\hline Red social & $\begin{array}{l}\text { Totalmente en } \\
\text { desacuerdo (1) }\end{array}$ & $\begin{array}{r}\text { En desacuerdo } \\
\text { (2) }\end{array}$ & $\begin{array}{r}\text { Neutral } \\
(3)\end{array}$ & $\begin{array}{r}\text { De acuerdo } \\
\text { (4) }\end{array}$ & $\begin{array}{r}\text { Totalmente de } \\
\text { acuerdo (5) }\end{array}$ & M & SD \\
\hline YouTube & $6(4.48 \%)$ & $4(2.99 \%)$ & $17(12.69 \%)$ & $50(37.31 \%)$ & $57(42.54 \%)$ & 4.10 & 1.03 \\
\hline Google + & $6(4.48 \%)$ & $5(3.73 \%)$ & $23(17.16 \%)$ & $58(43.28 \%)$ & $42(31.34 \%)$ & 3.93 & 1.02 \\
\hline Pinterest & $19(9.70 \%)$ & $9(6.72 \%)$ & $25(18.66 \%)$ & $50(37.31 \%)$ & $37(27.61 \%)$ & 3.66 & 1.22 \\
\hline Twitter & $19(14.18 \%)$ & $20(14.93 \%)$ & $24(17.91 \%)$ & $41(30.60 \%)$ & $30(22.39 \%)$ & 3.32 & 1.35 \\
\hline Flickr & $18(13.43 \%)$ & $12(8.96 \%)$ & $47(35.07 \%)$ & $36(26.87 \%)$ & $21(15.67 \%)$ & 3.22 & 1.21 \\
\hline Linkedln & $19(14.18 \%)$ & $13(9.70 \%)$ & $49(36.57 \%)$ & $32(23.88 \%)$ & $21(15.67 \%)$ & 3.17 & 1.22 \\
\hline Spotify & $24(17.91 \%)$ & $16(11.94 \%)$ & $50(37.31 \%)$ & $27(20.15 \%)$ & $17(12.69 \%)$ & 2.97 & 1.24 \\
\hline Whatsapp & $32(23.88 \%)$ & $32(23.88 \%)$ & $12(8.96 \%)$ & $41(30.60 \%)$ & $17(12.69 \%)$ & 2.84 & 1.41 \\
\hline Facebook & $33(24.63 \%)$ & $26(19.40 \%)$ & $24(17.91 \%)$ & $35(26.12 \%)$ & $16(11.94 \%)$ & 2.81 & 1.37 \\
\hline Instagram & $34(25.37 \%)$ & $37(27.61 \%)$ & $27(20.15 \%)$ & $23(17.16 \%)$ & $13(9.70 \%)$ & 2.58 & 1.29 \\
\hline
\end{tabular}

El smartphone desde la perspectiva docente: ¿una herramienta de tutorización o un catalizador de ciberacoso? Bernardo Tabuenca, Juan-José Sánchez-Peña y María-José Cuetos-Revuelta.

Página 6 de 14 
RED. Revista de Educación a Distancia. Núm. 59, Artíc. 01, 30-04-2019

DOI: http://dx.doi.org/10.6018/red/59/01

\begin{tabular}{|c|c|c|c|c|c|c|}
\hline Tuenti & $47(35.07 \%)$ & $35(26.12 \%)$ & $33(24.63 \%)$ & $12(8.96 \%)$ & $7(5.22 \%)$ & 2.23 \\
\hline
\end{tabular}

3.4. Riesgos de utilizar el móvil en los centros escolares.

En la cuarta pregunta de investigación se exploraba la valoración de los docentes acerca de los riesgos de utilizar el móvil en los centros escolares. Se implementó una escala de 5 elementos para que fueran comparables entre sí. Según se indica en la Tabla 4, los resultados revelan que los profesores dieron más importancia a unos riesgos que a otros identificando el Cyberbullying $(\mathrm{M}=4.43 ; \mathrm{SD}=0.68)$, la distracción y pérdida del foco de atención $(\mathrm{M}=3.98 ; \mathrm{SD}=1.00)$, y la dificultad para encontrar un consenso entre profesores sobre cómo utilizar el móvil $(\mathrm{M}=3.86 ; \mathrm{SD}=0.96)$ como los más relevantes. De los riesgos citados en la pregunta, los profesores dieron menor importancia al riesgo de dejar de lado actividades más tradicionales como la lectura o la expresión artística $(M=2.86 ; S D=1.23)$. La media total para los riesgos citados fue de $M=3.70(S D=1.13)$.

Tabla 4. Riesgos de utilizar el móvil en los centros escolares. $\mathrm{N}=135(\%)$

\begin{tabular}{|c|c|c|c|c|c|c|c|}
\hline Riesgos & $\begin{array}{r}\text { Totalmente en } \\
\text { desacuerdo (1) }\end{array}$ & $\begin{array}{r}\text { En } \\
\text { desacuerdo } \\
(2)\end{array}$ & $\begin{array}{r}\text { Neutral } \\
(3)\end{array}$ & $\begin{array}{r}\text { De } \\
\text { acuerdo } \\
(4)\end{array}$ & $\begin{array}{r}\text { Totalmente de } \\
\text { acuerdo (5) }\end{array}$ & $\mathbf{M}$ & SD \\
\hline $\begin{array}{l}\text { Cyberbullying (Acoso por móvil, } \\
\text { Internet...) }\end{array}$ & $1(0.75 \%)$ & $2(1.49 \%)$ & $3(2.24 \%)$ & $60(44.78 \%)$ & $68(50.75 \%)$ & 4.43 & 0.68 \\
\hline $\begin{array}{l}\text { Distracción y pérdida del foco de } \\
\text { atención. }\end{array}$ & $3(2.24 \%)$ & $13(9.70 \%)$ & $11(8.21 \%)$ & $63(47.01 \%)$ & $44(32.84 \%)$ & 3.98 & 1.00 \\
\hline $\begin{array}{l}\text { Dificultad para encontrar un } \\
\text { consenso entre profesores sobre } \\
\text { cómo utilizar el móvil. }\end{array}$ & $5(3.73 \%)$ & $6(4.48 \%)$ & $23(17.16 \%)$ & $68(50.75 \%)$ & $32(23.88 \%)$ & 3.86 & 0.96 \\
\hline Sobreexposición a Internet. & $6(4.48 \%)$ & $12(8.96 \%)$ & $37(27.61 \%)$ & $52(38.81 \%)$ & $27(20.15 \%)$ & 3.61 & 1.04 \\
\hline $\begin{array}{l}\text { Incompetencia digital del docente } \\
\text { (Destreza en el manejo de móviles y } \\
\text { aplicaciones). }\end{array}$ & $8(5.97 \%)$ & $24(17.91 \%)$ & $26(19.40 \%)$ & $55(41.04 \%)$ & $21(15.67 \%)$ & 3.42 & 1.13 \\
\hline $\begin{array}{l}\text { Dejar de lado actividades más } \\
\text { tradicionales, como la lectura o la } \\
\text { expresión artística. }\end{array}$ & $17(12.69 \%)$ & $45(33.58 \%)$ & $27(20.15 \%)$ & $29(21.64 \%)$ & $16(11.94 \%)$ & 2.86 & 1.23 \\
\hline
\end{tabular}

\subsection{Ventajas de utilizar el móvil en los centros escolares.}

En la quinta pregunta de investigación se explora cómo valoran los docentes las ventajas de utilizar el móvil en los centros escolares. Según se indica en la Tabla 5, los resultados revelan que los profesores dieron más importancia a unas ventajas que a otras identificando los juegos para atraer el interés del alumnado $(\mathrm{M}=4.44 ; \mathrm{SD}=0.71)$, potenciar la participación del alumnado $(\mathrm{M}=4.37 ; \mathrm{SD}=0.70)$, la adaptación al ritmo tecnológico de la sociedad $(\mathrm{M}=4.34 ; \mathrm{SD}=0.66)$, y la obtención de retroalimentación del alumnado $(\mathrm{M}=4.33 ; \mathrm{SD}=0.76)$ como las principales ventajas. De las ventajas citadas, los profesores dieron menor importancia a la comunicación entre profesor y alumno $(\mathrm{M}=3.93 ; \mathrm{SD}=1.02)$, a la identificación y denuncia de casos de acoso $(M=4.01 ; S D=0.81)$, y a la comunicación entre alumnos $(M=4.04 ; S D=0.95)$. La media total para las ventajas citadas fue de $M=4.21$ ( $S D=0.82)$.

El smartphone desde la perspectiva docente: ¿una herramienta de tutorización o un catalizador de ciberacoso? Bernardo Tabuenca, Juan-José Sánchez-Peña y María-José Cuetos-Revuelta.

Página 7 de 14 
RED. Revista de Educación a Distancia. Núm. 59, Artíc. 01, 30-04-2019

DOI: http://dx.doi.org/10.6018/red/59/01

Tabla 5. Ventajas de utilizar móvil en los centros escolares. $\mathrm{N}=135(\%)$

\begin{tabular}{|c|c|c|c|c|c|c|c|}
\hline Ventajas & $\begin{array}{l}\text { Totalmente en } \\
\text { desacuerdo (1) }\end{array}$ & $\begin{array}{c}\text { En } \\
\text { desacuerdo } \\
(2) \\
\end{array}$ & $\begin{array}{l}\text { Neutral } \\
\text { (3) }\end{array}$ & $\begin{array}{r}\text { De } \\
\begin{array}{c}\text { acuerdo } \\
(4)\end{array} \\
\end{array}$ & $\begin{array}{l}\text { Totalmente de } \\
\text { acuerdo (5) }\end{array}$ & $\mathbf{M}$ & SD \\
\hline $\begin{array}{l}\text { Juegos para atraer el interés del } \\
\text { alumnado. }\end{array}$ & $1(0.75 \%)$ & $1(0.75 \%)$ & $8(5.97 \%)$ & $52(38.81 \%)$ & $72(53.72 \%)$ & 4.44 & 0.71 \\
\hline $\begin{array}{l}\text { Potenciar la participación del } \\
\text { alumnado. }\end{array}$ & $1(0.75 \%)$ & $1(0.75 \%)$ & $8(5.97 \%)$ & $61(45.52 \%)$ & $63(47.01 \%)$ & 4.37 & 0.70 \\
\hline $\begin{array}{l}\text { Adaptación a la sociedad y ritmo } \\
\text { tecnológico. }\end{array}$ & $1(0.75 \%)$ & $1(0.75 \%)$ & $5(3.73 \%)$ & $71(52.99 \%)$ & $56(41.79 \%)$ & 4.34 & 0.66 \\
\hline $\begin{array}{l}\text { Tests para obtener } \\
\text { retroalimentación del alumnado. }\end{array}$ & $0(0 \%)$ & $5(3.73 \%)$ & $9(6.72 \%)$ & $56(41.79 \%)$ & $64(47.76 \%)$ & 4.33 & 0.76 \\
\hline $\begin{array}{l}\text { Fines pedagógicos: diccionario, } \\
\text { calculadora, brújula u otras } \\
\text { aplicaciones. }\end{array}$ & $1(0.75 \%)$ & $5(3.73 \%)$ & $14(10.45 \%)$ & $54(40.30 \%)$ & $60(44.78 \%)$ & 4.24 & 0.84 \\
\hline $\begin{array}{l}\text { Personalización del aprendizaje } \\
\text { por parte del alumno. }\end{array}$ & $1(0.75 \%)$ & $3(2.24 \%)$ & $14(10.45 \%)$ & $62(46.27 \%)$ & $54(40.30 \%)$ & 4.23 & 0.78 \\
\hline Compartir buenas prácticas. & $1(0.75 \%)$ & $2(1.49 \%)$ & $17(12.69 \%)$ & $61(45.52 \%)$ & $53(39.55 \%)$ & 4.21 & 0.78 \\
\hline Planificación de tareas. & $1(0.75 \%)$ & $5(3.73 \%)$ & $13(9.70 \%)$ & $65(48.51 \%)$ & $50(37.31 \%)$ & 4.18 & 0.81 \\
\hline Comunicación entre alumnos. & $3(2.24 \%)$ & $8(5.97 \%)$ & $16(11.94 \%)$ & $61(45.52 \%)$ & $46(34.33 \%)$ & 4.04 & 0.95 \\
\hline $\begin{array}{l}\text { Identificar y denunciar casos de } \\
\text { acoso. }\end{array}$ & $0(00 \%)$ & $5(3.73 \%)$ & $28(20.90 \%)$ & $62(46.27 \%)$ & $39(29.10 \%)$ & 4.01 & 0.81 \\
\hline $\begin{array}{l}\text { Comunicación entre profesor y } \\
\text { alumno. }\end{array}$ & $4(2.99 \%)$ & $11(8.21 \%)$ & $17(12.69 \%)$ & $60(44.78 \%)$ & $42(31.34 \%)$ & 3.93 & 1.02 \\
\hline
\end{tabular}

\subsection{Regulación del uso de los dispositivos móviles en los centros escolares}

En la sexta pregunta de investigación se pretendía concretar qué regulación existe actualmente en los centros educativos españoles con respecto al uso de dispositivos móviles y además explorar la opinión de los docentes acerca de qué figura debe regular el uso de dispositivos móviles en el contexto escolar.

Los resultados reportados en la Tabla 6 indican que el uso del móvil está prohibido al alumnado en un $55.22 \%$ mientras que sólo un $5.96 \%$ prohíbe el uso a profesores y alumnos. En cuanto a los centros más livianos en lo que se refiere a limitaciones, existe un $7.46 \%$ en los que no hay restricciones al respecto, mientras que para un $12.69 \%$ está permitido el uso del dispositivo móvil en los descansos.

En lo que respecta a la figura que debe regular el uso de dispositivos móviles, la gran mayoría piensa que debe estar regulada, siendo la opción del centro escolar la más apropiada para un $50.75 \%$ seguida del Ministerio de Educación para un $26.12 \%$.

Tabla 6. Regulación del uso de dispositivos móviles en los centros educativos.

\begin{tabular}{lrr} 
¿Qué regulación existe actualmente con respecto al uso de dispositivos móviles en los centros educativos? \\
\hline
\end{tabular}

¿Qué figura debería regular el uso de los dispositivos móviles en los centros educativos?

\begin{tabular}{lrr}
\hline & $\mathrm{N}=134$ & $\%$ \\
Centro escolar & 68 & 50.75 \\
Ministerio de Educación & 35 & 26.12 \\
Profesor & 16 & 11.94 \\
Comunidad autónoma & 12 & 8.96 \\
No debe estar regulado & 1 & 0.75 \\
NS / NC & 2 & 1.49 \\
\hline
\end{tabular}

El smartphone desde la perspectiva docente: ¿una herramienta de tutorización o un catalizador de ciberacoso? Bernardo Tabuenca, Juan-José Sánchez-Peña y María-José Cuetos-Revuelta. 
RED. Revista de Educación a Distancia. Núm. 59, Artíc. 01, 30-04-2019

DOI: http://dx.doi.org/10.6018/red/59/01

3.7. Edad apropiada para el uso de dispositivos móviles desde la perspectiva docente.

En la séptima pregunta de investigación se pretendía concretar la edad apropiada para entregar un móvil con Internet a un niño desde la perspectiva docente.

La Figura 3 representa la edad de los profesores que participaron en el estudio, en contraste con la edad que consideraron apropiada para entregar un móvil con Internet a un niño. La edad media señalada por los profesores para entregar un móvil con Internet a un niño resultó de 14 años, con una dispersión intercuartílica de 3 años. Las mujeres reportaron ligeramente por debajo (14.21) con respecto a los hombres (13.85). Las diferencias clasificadas por Comunidad Autónoma y tipo de docencia impartida (primaria, secundaria, etc.) no fueron significativas. Los profesores participantes tenían una media de 32 años, habiendo una dispersión intercuartílica de 7 años.

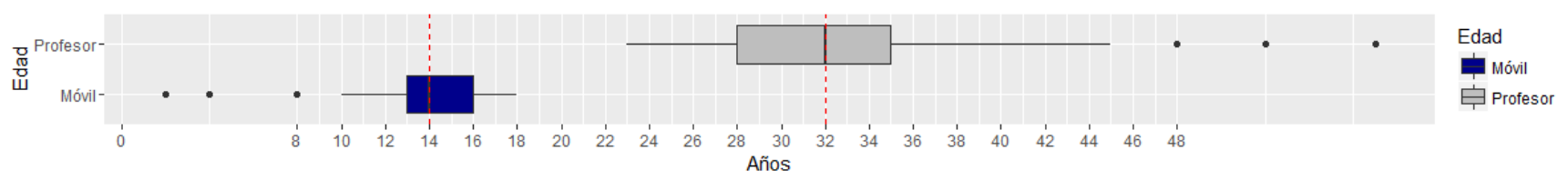

Figura 3. Edad apropiada para entregar un móvil con Internet a un niño desde la perspectiva docente.

\subsection{Estudio de correlación}

Se realizó un estudio de correlación con el objetivo de explorar si existe relación entre la edad de los profesores con sus valoraciones acerca de 1) los riesgos de utilizar dispositivos móviles en el aula, 2) las ventajas de utilizar móviles en el aula, y 3) la edad apropiada para entregar un teléfono móvil con Internet a un niño. El análisis de correlación de Pearson determinó los resultados que se muestran en la Tabla 7. El estudio de correlación de Pearson proporciona una medida $(r)$ con la fortaleza de la relación lineal entre dos variables cuyos valores oscilan entre $-1<0<1$. Los valores más cercanos a los extremos $10-1$ representan una correlación positiva o negativa respectivamente más fuerte, lo que significa que la segunda variable tiende a aumentar o disminuir cuando los valores del primer valor se incrementan y viceversa. Cuanto más cerca estén los valores de 0 , más débil será la correlación. Un valor $p$ menor que 0.01 se toma como indicador de correlaciones significativas. Podemos describir verbalmente la fortaleza de la correlación usando la guía que sugiere Evans (1996) para el valor absoluto de r (Fuerza: 0-0.19 "Muy débil"; 0.2-0.39 "Débil"; 0.4-0.59 "Moderada"; 0.6-0.79 " Fuerte "; 0.8-1" Muy fuerte ").

Tabla 7. Resultados del análisis de correlación de Pearson.

\begin{tabular}{r|cccc}
$r$ & Edad Profesor & Redes sociales & Riesgos móvil & Ventajas móvil \\
\hline Edad Profesor & 1 & - & - & - \\
Redes sociales & 0.06 & 1 & - & - \\
Riesgos móvil & -0.10 & 0.05 & 1 & - \\
Ventajas móvil & $-0.22^{*}$ & $0.26^{*}$ & 0.09 & 1 \\
\hline
\end{tabular}

Los resultados del análisis sugieren una correlación negativa débil entre la edad del profesor y su percepción acerca de las ventajas de utilizar dispositivos móviles $(r=-0.22, p=0.011)$. Como se ilustra en la Figura 4, cuanto mayor es la edad de los profesores, menos espléndidos son en calificar las ventajas del uso de dispositivos móviles en el aula. Sin embargo, no existe correlación entre la edad del profesor y su valoración acerca de los riesgos de utilizar móviles $(r=-0.10, p=0.25)$. Además, los resultados del análisis muestran la no existencia de correlación entre la edad del profesor y su predisposición a utilizar las redes sociales $(r=0.06, p=0.46)$. Sin embargo, el análisis identificó una correlación positiva débil entre la predisposición a utilizar la redes sociales y su valoración acerca de las ventajas de utilizar dispositivos móviles en el aula ( $r=0.26, p=0.0024)$.

El smartphone desde la perspectiva docente: ¿una herramienta de tutorización o un catalizador de ciberacoso? Bernardo Tabuenca, Juan-José Sánchez-Peña y María-José Cuetos-Revuelta.

Página 9 de 14 
RED. Revista de Educación a Distancia. Núm. 59, Artíc. 01, 30-04-2019

DOI: http://dx.doi.org/10.6018/red/59/01

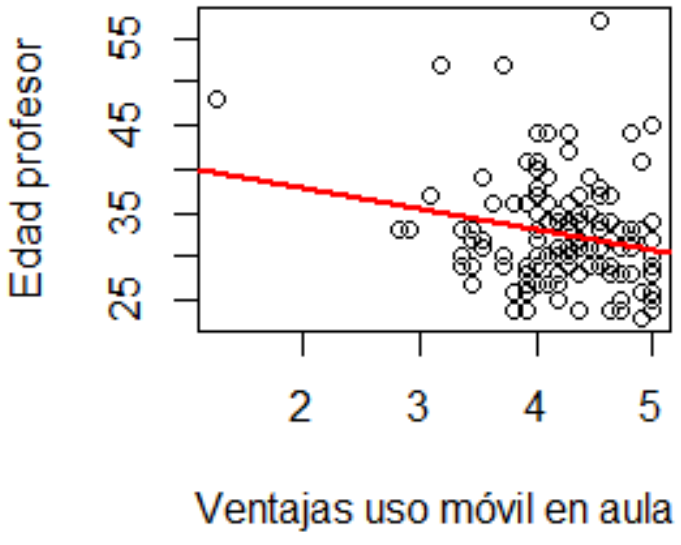

a) Cuanto mayores son los profesores, menos ventaja observan en utilizar móviles en el aula

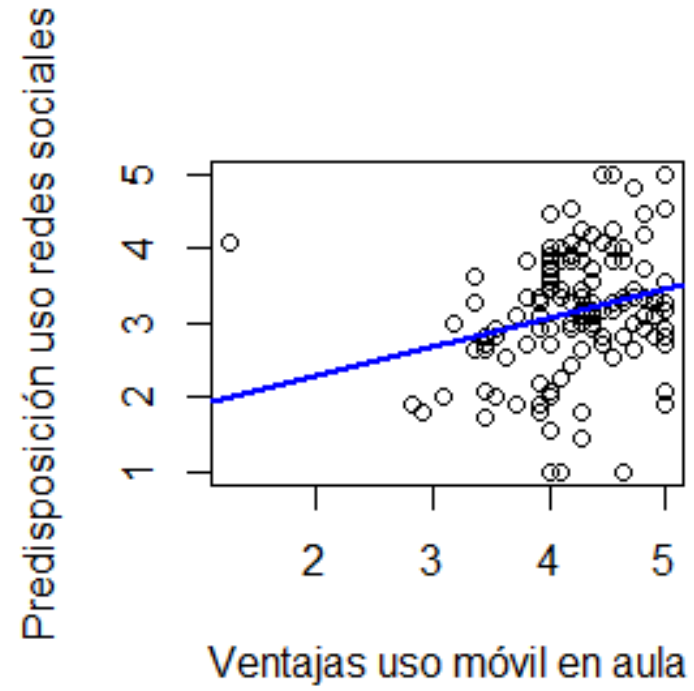

a) Cuanto más predispuestos están los profesores a utilizar redes sociales, más ventaja observan en utilizar móviles en el aula

Figura 4. Estudio de correlación de Pearson

\section{Discusión y conclusiones}

Los resultados de este trabajo han permitido poner en contraste los riesgos y oportunidades del uso de dispositivos en los centros educativos desde la perspectiva docente.

Los profesores utilizan de forma mayoritaria los dispositivos móviles para su actividad docente (77.42\% móvil y $51 \%$ tableta digital), principalmente para tareas de gestión, control de asistencia, compartir documentos, comunicarse con compañeros (y familias), gestión de contenidos y para introducir elementos de ludificación con herramientas móviles como Kahoot, Idoceo, Edmodo, Socrative, ClassDojo, o iSeneca (Figura 1). En futuras investigaciones, sería de interés explorar qué riesgos y oportunidades conlleva el uso de dispositivos en padres de alumnos.

La mayoría de los profesores están predispuestos a utilizar dispositivos móviles propios o corporativos como herramienta para tutorizar a los alumnos (Figura 2). Sin embargo, sigue habiendo un notable porcentaje de profesores que no están dispuestos a utilizar su dispositivo móvil personal para tutorizar alumnos (27.61\%), porcentaje que se reduce hasta el $4.47 \%$ si los móviles utilizados fueran corporativos. Independientemente del dispositivo móvil que se utilice, la opción más popularmente aceptada es que el móvil se utilice exclusivamente en horario laboral y periodo lectivo. Existen dos diferencias notables entre el uso de dispositivos móviles propios y no propios (Tabuenca, 2015): 1) competencia digital, los usuarios ven mermada su competencia digital al realizar tareas de aprendizaje sobre dispositivos móviles no propios; 2) privacidad, muchos usuarios piensan que su móvil pertenece a su ámbito personal y son reacios a introducirlo en el ámbito profesional. Estos resultados sugieren el uso del móvil corporativo como facilitador para tutorizar al alumnado. Sería de interés para futuros estudios investigar las variables de privacidad y competencia digital, así como identificar las razones por las que algunos profesores (4.47\%) no están dispuestos a utilizarlo en ningún caso.

Las redes sociales son un elemento de comunicación frecuente entre estudiantes. En este trabajo se pretendía identificar cuáles son las redes sociales que los docentes estarían dispuestos a utilizar como canal para comunicarse con los alumnos. En la actualidad, las sesiones lectivas tradicionales se acompañan ocasionalmente de contenidos multimedia. Por esta razón YouTube y Pinterest estuvieron entre los mejor valorados (Tabla 3). Google+ también fue bien valorado por su compatibilidad con otras herramientas ofimáticas como Google Drive que facilitan la compartición y acceso a contenidos, o Google Classroom que permite

El smartphone desde la perspectiva docente: ¿una herramienta de tutorización o un catalizador de ciberacoso? Bernardo Tabuenca, Juan-José Sánchez-Peña y María-José Cuetos-Revuelta.

Página 10 de 14 
RED. Revista de Educación a Distancia. Núm. 59, Artíc. 01, 30-04-2019

DOI: http://dx.doi.org/10.6018/red/59/01

creación de aulas virtuales, distribuir tareas y evaluar contenidos. Tuenti, Instagram, Facebook y WhatsApp fueron las peor valoradas. Los profesores pasan muchas horas cara a cara con el alumnado y quizás no sea necesario suplantar esta comunicación con otra menos directa y que pueda llevar a ambigüedades. Llama la atención que cuando a los profesores se les preguntó acerca de las ventajas de utilizar el dispositivo móvil, la "Comunicación entre profesor y alumno" obtuvo la menor puntuación entre los elementos listados (Ver Tabla 5). El uso de las redes sociales en el ámbito educativo debe considerarse con mucha cautela ya que ninguna de las redes sociales citadas admite registrar usuarios menores de 16 años. En futuras investigaciones sería de interés identificar qué características concretas resultan de interés a los profesores en cada red social, esto es, compartición de videos, fotografías, intercambio de mensajes de texto, valoración de comentarios ("Me gusta", etiquetar como "favorito", ...) y con qué fin.

El ciberacoso es el principal riesgo de utilizar móviles en los centros educativos españoles (Tabla 4). Los resultados de este estudio contrastan con estudios anteriores en otros países donde el ciberacoso no es el principal riesgo identificado por los docentes (O'Bannon \& Thomas, 2015; Eden, Heiman, \& Olenik-Shemesh, 2013; Ryan, Kariuki, \& Yilmaz, 2011; Huang \& Chou, 2013; Li, 2008). La "distracción y pérdida del foco de atención" que producen los móviles a los alumnos en los centros educativos fue señalada como segundo riesgo en importancia. Los móviles tienen instaladas múltiples aplicaciones que requieren la atención del usuario con notificaciones de diferentes grados de urgencia y que provocan la dispersión. En tercer lugar se valoró la "dificultad para encontrar un consenso entre profesores sobre cómo utilizar el móvil" lo que pone de manifiesto las diferencias entre docentes para utilizar el móvil: algunas personas responden inmediatamente a los correos electrónicos mientras otros lo hacen una vez al comenzar el día u otro determinado patrón. Establecer directrices sobré cómo se debe utilizar el móvil en lo educativo y que los profesores lleguen a un consenso es un campo que debe explorarse en futuras investigaciones, para identificar qué puntos generan más controversia y poder aliviarlos con tecnología u otras soluciones.

Las ventajas de utilizar móviles en el aula fueron mejor valoradas que los riesgos $(M=4.21>3.71)$. Los juegos móviles orientados a atraer el interés del alumnado y potenciar su participación son la utilidad mejor valorada por los docentes (Tabla 5). La ludificación (del inglés "gamification") consiste en la introducción de recompensas y penalizaciones mediante juegos que pretenden divertir a los alumnos a la vez que les motiva a realizar acciones orientadas a la consecución del aprendizaje (Calvo-Morata \& Fernández-Manjón, 2018). Existen diferentes revisiones de la literatura listando los beneficios de los juegos en la educación (Connolly, Boyle, Macarthur, Hainey, \& Boyle, 2012; Michael \& Chen, 2005). En lo que respecta al ciberacoso, para futuras investigaciones sería de interés explorar experiencias con juegos móviles para concienciar, y además denunciar casos de ciberacoso. Variables como la privacidad del denunciante, o el formato de la denuncia (mensaje de texto, fotografía o video), deben tomarse en cuenta en futuros estudios.

Las medidas legales frente al ciberacoso cada vez son más demandadas y aunque en el $92 \%$ de los centros examinados en este estudio tiene alguna restricción en cuanto al uso de móviles, no existe consenso sobre qué organismo debería regular su uso. La mayoría de los docentes participantes en este estudio (50.75\%) reportaron que debería ser cada centro escolar quien establezca la manera de regular su uso (Tabla 6). Aunque difícilmente habría consenso dentro de los propios centros, este tipo de soluciones permitiría ajustar la norma a la predisposición de la mayoría de los profesores, o adaptarla a periodos temporales en los que pudiera haber más o menos conflicto. Sin embargo, las iniciativas de llaneros solitarios (Bates, 2000) en las que cada profesor se adentra en su uso mediante tareas de evaluación (idoceo, google classroom), colaborativas (google drive), u obtención de retroalimentación del alumnado (karhoot, socrative) con herramientas como las que se ilustran en la figura 1, fueron secundadas por sólo un $12.69 \%$. El $26.12 \%$ tuvo una visión más generalista señalando que debería ser el Ministerio de Educación quien lo regulara y así desechar divergencias entre profesores, centros y comunidades autónomas.

Los profesores participantes en este estudio indicaron que la edad media apropiada para entregar un móvil con Internet a un niño es de 14 años (ver figura 3). Estas conclusiones distan notablemente de la realidad (INE, 2016) en la que el $51 \%$ de los niños españoles de 11 años ya dispone de un móvil con Internet. Los padres parecen estar dando prioridad a la necesidad social de sus hijos, o la agilidad de tenerlos a un whatsapp de distancia, o la tranquilidad que dan los dispositivos a los padres manteniendo a sus hijos entretenidos. Esta discrepancia entre edades debe tomarse en consideración. Los años de diferencia en la adolescencia son

El smartphone desde la perspectiva docente: ¿una herramienta de tutorización o un catalizador de ciberacoso? Bernardo Tabuenca, Juan-José Sánchez-Peña y María-José Cuetos-Revuelta.

Página 11 de 14 
RED. Revista de Educación a Distancia. Núm. 59, Artíc. 01, 30-04-2019

DOI: http://dx.doi.org/10.6018/red/59/01

especialmente largos en el mundo virtual porque las interacciones ocurren a un ritmo trepidante, y en un momento en el que los niños son extraordinariamente sensibles al acoso.

Los profesores más jóvenes fueron más espléndidos valorando las ventajas de utilizar dispositivos móviles en el aula (Ver tabla 2 y figura 4). Asimismo, parece haber una relación entre las valoraciones positivas acerca del uso del móvil en el aula, que también crecieron con la predisposición de los profesores a utilizar redes sociales para comunicarse con lo alumnos. Esta conclusiones deben considerarse con cautela ya que el índice de correlación es muy débil $(r<0.39)$. Debe tenerse en cuenta que los profesores participantes en este estudio pudieran tener una competencia digital por encima de la media por tres razones: 1) su media de edad es de 32 años (ver figura 3); 2) son alumnos cursando un máster universitario online; 3) el máster es de la especialidad de tecnología educativa. En futuros estudios, sería de interés explorar ventajas, riesgos y edad apropiada para entregar el móvil a un niño desde la perspectiva de docentes con una competencia digital media.

Este trabajo ha identificado claves sobre qué uso se hace del Smartphone con fines educativos en la era móvil, cuál es la predisposición docente a utilizar móviles propios y redes sociales como herramienta de tutorización, qué amenazas y ventajas se identifican de su uso, qué regulación existe actualmente con respecto al uso del móvil en los centros educativos y qué edad se considera apropiada para entregar un móvil con internet a un niño/adolescente. Las conclusiones extraídas en este trabajo deben tomarse con cautela ya que el alcance y potencialidades de los modelos sobre el aprendizaje en entornos conectados y ubicuos está aún por madurar (Zapata, 2015).

Presentación del artículo: 11 de diciembre de 2018

Fecha de aprobación: 1 de febrero de 2019

Fecha de publicación: 30 de abril de 2019

Tabuenca, B., Sánchez-Peña, J.J, y Cuetos-Revuelta, M.J. (2019). El smartphone desde la perspectiva docente: ¿una herramienta de tutorización o un catalizador de ciberacoso? RED. Revista de Educación a Distancia, 59. DOI http://dx.doi.org/10.6018/red/59/01

\section{Financiación}

Esta investigación no ha recibido ninguna subvención específica de los organismos de financiación en los sectores públicos, comerciales o sin fines de lucro.

\section{Agradecimientos}

Los autores de este trabajo agradecen a Bureau Veritas Formación y a sus profesores haber facilitado esta investigación dentro del Máster Oficial Universitario en Tecnología Educativa.

\section{Referencias}

Adam Becker, S., Cummins, M., Davis, A., Freeman, A., Hall Gieseinger, C., \& Ananthanarayanan, V. (2017). NMC Horizon Report Higher Education Edition 2017. Reading. https://doi.org/ISBN 978-0-9977215-7-7

ANAR, F., \& Mutua-Madrileña, F. (2017). Il Estudio sobre acoso escolar y ciberbullying según los afectados. Madrid. Retrieved from https://www.anar.org/wp-content/uploads/2017/04/INFORME-II-ESTUDIOCIBERBULLYING.pdf

Brazuelo-Grund, F., Gallego-Gil, D. J., \& Cacheiro-González, M. L. (2017). Los docentes ante la integración educativa del teléfono móvil en el aula. Revista de Educación a Distancia (RED), 52(52), 30-2017. https://doi.org/10.6018/red/52/6

Calvo-Morata, A., \& Fernández-Manjón, B. (2018). Conectado: concienciando contra el cyberbullying mediante un videojuego educativo. Didáctica, Innovación y Multimedia, (36).

El smartphone desde la perspectiva docente: ¿una herramienta de tutorización o un catalizador de ciberacoso? Bernardo Tabuenca, Juan-José Sánchez-Peña y María-José Cuetos-Revuelta.

Página 12 de 14 
RED. Revista de Educación a Distancia. Núm. 59, Artíc. 01, 30-04-2019

DOI: http://dx.doi.org/10.6018/red/59/01

Cerezo-Ramirez, F. (1997). Conductas agresivas en la edad escolar (Piramide). Madrid.

Cerezo-Ramirez, F. (2012). Psique : Bullying a través de las TIC. Boletín Científico Sapiens Research, 2(2), 2429.

Cerezo Ramírez, F., \& Rubio-Hernández, F.-J. (2017). Medidas relativas al acoso escolar y ciberacoso en la normativa autonómica española. Un estudio comparativo. Revista Electrónica Interuniversitaria de Formación Del Profesorado, 20(1), 113-126. https://doi.org/http://dx.doi.org/10.6018/reifop.20.1.253391

Connolly, T. M., Boyle, E. A., Macarthur, E., Hainey, T., \& Boyle, J. M. (2012). A systematic literature review of empirical evidence on computer games and serious games. Computers \& Education. https://doi.org/10.1016/j.compedu.2012.03.004

Eden, S., Heiman, T., \& Olenik-Shemesh, D. (2013). Teachers' perceptions, beliefs and concerns about cyberbullying. British Journal of Educational Technology, 44(6), 1036-1052. https://doi.org/10.1111/j.14678535.2012.01363.x

Garaigordobil, M. (2011). Prevalencia y consecuencias del cyberbullying: Una revisión. International Journal of Psychology and Psychological Therapy.

Garmendia Larrañaga, M., Jiménez Iglesias, E., Casado, M. Á., \& Marcheroni, G. (2016). Net Children Go Mobile: Riesgos y oportunidades en internet y uso de dispositivos móviles entre menores españoles (20102015). Madrid. Retrieved from https://www.ehu.eus/documents/1370232/0/Informe+NET+CHILDREN+RED.ES+España+2016.pdf/62625 $1 \mathrm{bc}-\mathrm{f} 3 \mathrm{f} 1-47 \mathrm{ee}-\mathrm{a} 06 \mathrm{~b}-8401 \mathrm{~b} 91352 \mathrm{df}$

Giménez-Gualdo, A., Maquilón-Sánchez, J. J., \& Arnaiz Sánchez, P. (2014). Acceso a las tecnologías, rendimiento académico y cyberbullying en escolares de secundaria. Revista Iberoamericana de Psicología Y Salud, 5(2), 119-133. Retrieved from http://dialnet.unirioja.es/servlet/articulo?codigo=4762637

Hosseinmardi, H., Rafiq, R. I., Han, R., Lv, Q., \& Mishra, S. (2016). Prediction of cyberbullying incidents in a media-based social network. In Proceedings of the 2016 IEEE/ACM International Conference on Advances in Social Networks Analysis and Mining, ASONAM 2016 (pp. 186-192). https://doi.org/10.1109/ASONAM.2016.7752233

Huang, Y. Y., \& Chou, C. (2013). Revisiting cyberbullying: Perspectives from Taiwanese teachers. Computers and Education, 63, 227-239. https://doi.org/10.1016/j.compedu.2012.11.023

INE. (2016). Encuesta sobre Equipamiento y Uso de Tecnologías de Información y Comunicación en los Hogares. Madrid. Retrieved from http://www.ine.es/prensa/np991.pdf

Johnson, L., Adams Becker, S., Estrada, V., \& Freeman, A. (2015). NMC Horizon Report: 2015 K-12 Edition. Austin, Texas.

Kukulska-Hulme, A., \& Traxler, J. (2005). Mobile learning: A handbook for educators and trainers. Mobile Learning: A Handbook for Educators and Trainers. https://doi.org/10.4324/9780203003428

$\mathrm{Li}, \mathrm{Q}$. (2008). Cyberbullying in schools: An examination of preservice teachers' perception. Canadian Journal of Learning and Technology, 34(2). Retrieved from http://www.cjlt.ca/index.php/cjlt/article/view/494/225

Likert, R. (1932). A technique for the measurement of attitudes. Archives of Psychology. https://doi.org/2731047

Mercedes Durán, R. M.-P. (2015). Ciberacoso mediante teléfono móvil e Internet en las relaciones de noviazgo entre jóvenes Cyberbullying trough Mobile Phone and the Internet in Dating Relationships among Youth People. Comunicar, 22(44), 159-167. https://doi.org/10.3916/C44-2015-17

Michael, D. R., \& Chen, S. L. (2005). Serious Games: Games That Educate, Train, and Inform. Education. https://doi.org/10.1021/la104669k

O'Bannon, B. W., \& Thomas, K. M. (2015). Mobile phones in the classroom: Preservice teachers answer the call. Computers and Education, 85, 110-122. https://doi.org/10.1016/j.compedu.2015.02.010

Ortega Barón, Jessica; Buelga, Sofía; Cava, M. J. (2016). Influencia del clima escolar y familiar en adolescentes , víctimas de ciberacoso. Comunicar, 24, 57-65. https://doi.org/10.3916/C46-2016-06

Prodócimo, E., Cerezo, F., \& Arense, J. J. (2014). Acoso escolar: Variables sociofamiliares como factores de riesgo o de protección. Behavioral Psychology/ Psicologia Conductual, 22(2), 345-359.

Ryan, T., Kariuki, M., \& Yilmaz, H. (2011). A comparative analysis of cyberbullying perceptions of preservice educators: Canada and Turkey. Turkish Online Journal of Educational Technology, 10(3), 1-12.

Sharples, M., Taylor, J., \& Vavoula, G. (2007). A Theory of Learning for the Mobile Age. Learning. https://doi.org/10.4135/9781848607859

Smith, P. K., Mahdavi, J., Carvalho, M., Fisher, S., Russell, S., \& Tippett, N. (2008). Cyberbullying: Its nature and impact in secondary school pupils. Journal of Child Psychology and Psychiatry and Allied Disciplines, 49(4), 376-385. https://doi.org/10.1111/j.1469-7610.2007.01846.x

El smartphone desde la perspectiva docente: ¿una herramienta de tutorización o un catalizador de ciberacoso? Bernardo Tabuenca, Juan-José Sánchez-Peña y María-José Cuetos-Revuelta.

Página 13 de 14 
RED. Revista de Educación a Distancia. Núm. 59, Artíc. 01, 30-04-2019

DOI: http://dx.doi.org/10.6018/red/59/01

Tabuenca, B., Drachsler, H., Ternier, S., \& Specht, M. (2012). OER in the Mobile Era: Content Repositories' Features for Mobile Devices and Future Trends. ELearning Papers, 32(Special Issue on Mobile Learning), 16. Retrieved from http://dspace.learningnetworks.org/handle/1820/4656

Tabuenca, B., Kalz, M., Drachsler, H., \& Specht, M. (2015). Time will tell: The role of mobile learning analytics in self-regulated learning. Computers \& Education, 89, 53-74. https://doi.org/10.1016/j.compedu.2015.08.004

Tabuenca, B., Ternier, S., \& Specht, M. (2013). Patrones cotidianos en estudiantes de formación continua para la creación de ecologías de aprendizaje. Revista de Educación a Distancia.

Tabuenca, B., Verpoorten, D., \& Specht, M. (2013). Fomento de la práctica reflexiva sobre el aprendizaje mediante el uso de tecnologías móviles. Revista de Educación a Distancia, 37, 1-14.

Zapata, M. (2015). Teorías y modelos sobre el aprendizaje en entornos conectados y ubicuos. Education in the Knowledge Society (Eks). https://doi.org/10.14201/eks201516169102

El smartphone desde la perspectiva docente: ¿una herramienta de tutorización o un catalizador de ciberacoso? Bernardo Tabuenca, Juan-José Sánchez-Peña y María-José Cuetos-Revuelta.

Página 14 de 14 